\title{
Transabdominal Ultrasound
}

National Cancer Institute

\section{Source}

National Cancer Institute. Transabdominal Ultrasound. NCI Thesaurus. Code C71375.

A method of ultrasound imaging in which the ultrasound probe is pressed against the skin of the abdomen in order to create an image of the abdominal organs. 